\title{
Highly Accurate Prediction of Jobs Runtime Classes
}

\author{
Anat Reiner-Benaim \\ Department of Statistics \\ University of Haifa \\ Haifa, Israel
}

\author{
Anna Grabarnick \\ Department of Statistics \\ University of Haifa \\ Haifa, Israel
}

\author{
Edi Shmueli \\ Intel Corporation \\ Haifa, \\ Israel
}

\begin{abstract}
Separating the short jobs from the long is a known technique to improve scheduling performance. This paper describes a method developed for accurately predicting the runtimes classes of the jobs to enable the separation. Our method uses the fact that the runtimes can be represented as a mixture of overlapping Gaussian distributions, in order to train a CART classifier to provide the prediction. The threshold that separates the short jobs from the long jobs is determined during the evaluation of the classifier to maximize prediction accuracy. The results indicate overall accuracy of $\mathbf{9 0 \%}$ for the data set used in the study, with sensitivity and specificity both above $90 \%$.
\end{abstract}

Keywords-Runtime Prediction; Job Scheduler; Server Farms; Classifier; Mixture Distribution

\section{INTRODUCTION}

Supplying job schedulers with information on how long the jobs are expected to run enabled the development of the backfilling algorithms, which leverage the information to pack the jobs more efficiently and improve system utilization [1]. The backfilling algorithms, however, were designed for parallel systems, in which the jobs require many processors in order to execute, and processor fragmentation (idleness) is a big concern. Thus in parallel system environments the scheduler needs to know the actual runtimes of the jobs (use numeric predictions) to be able to optimize the schedule and improve performance [10].

Our work targets systems in which most jobs are serial, like server farms that are used for software testing. In serial system environments sophisticated scheduling algorithms are not required, and in order to improve performance it is enough to simply separate the short jobs from the long ones, and assign them to different queues in the system [12]. The separation reduces the likelihood that short jobs will be delayed after long ones, improves the average turn-around times of the jobs and overall system throughput (Figure 1).

Respectively, to implement such a system it is enough to only predict the runtime classes of the jobs - whether they will be short or long, in order to assign them to the right queue. On the other hand, any misclassification of the jobs can severely impact performance. For example, mistakenly assigning long jobs to the short jobs queue will cause many of the short jobs to be delayed, average turnaround time to increase, and the overall throughput to decrease as a result.

Motivated by the later usage model (server farms), a method that allows predicting the runtime classes of the jobs with high accuracy was developed.

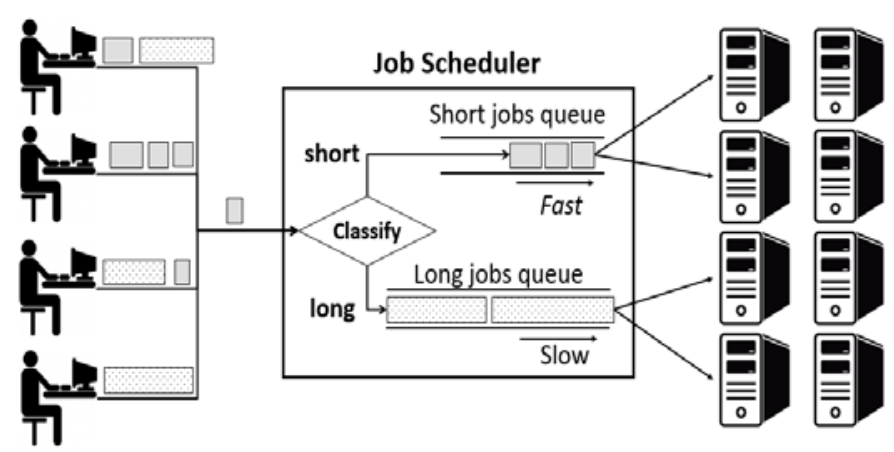

Fig. 1. Separating the short jobs from the long reduces the likelihood that short jobs will be delayed after long ones and improves system performance

The method is based on applying a log transformation on the runtimes of the jobs (historical records), revealing a mixture of two overlapping Gaussian distributions that represent the short and long jobs. We use the mixture model to determine the distribution parameters and to set the initial separation threshold between the short and long runtime populations.

A key design aspect for the proposed method is to be able to predict the classes with high accuracy. In order to achieve high accuracy, the threshold that separates the short jobs from the long is not determined in advance (which can lead to an eventual high misclassification rate). Instead, the threshold is determined as part of the evaluation of the classifier: a subset of the data that is close to the means of the distributions is used for training the classifier, and then the full dataset is used to select the threshold that optimizes a desired target function.

For class prediction for newly incoming jobs, the CART classifier is used [18]. CART is suitable for binary classification and can account for both continuous and categorical classifying variables, and is based on a tree optimizing algorithm that minimizes classification error while reducing overfitting by branch pruning.

The proposed method was applied on a job trace obtained from one of Intel's server farms, and which contained more than one million job records. Setting the target on achieving the best trade-off between misclassifications of short jobs and misclassifications of long jobs resulted in prediction accuracy of $90 \%$ (total misclassification rate of $10 \%$ ) on the independent validation set. The predictions were based on estimated distribution means of 140 and 3,500 seconds for the "short" and "long" classes, respectively, and a separating threshold of 608 seconds. 
This paper is organized as follows. Section 2 describes the data that is used for training, testing and validating the model. Section 3 describes the initial class labeling based on the mixture model analysis. Section 4 reviews the CART model. Section 5 describes the learning algorithm along with the optimal threshold determination procedure for best accuracy. Section 6 describes the results of the study. Section 7 surveys related work and Section 8 concludes the paper.

\section{THE DATA}

Our data is based on two traces obtained from one of Intel's server farms. The first trace, which was used to train the model, contained a sample of around one million job records that executed in the farm during a period of ten consecutive days. The second trace, which was used to validate the model, contained a sample of additional 755,000 records (approximately) of jobs that executed during a period of seven consecutive days. The validation on independent data is important for establishing the robustness of the model obtained in the training stage.

Each record in the traces contained 13 fields pertaining to a particular job. The continuous variables: "Submitime", "Starttime" and "Finishtime" indicated when the job was submitted, when the job started, and when the job finished executing, respectively. In order not to reveal any information about the workload, the traces did not contain any descriptive information about the jobs. Instead, the values in the fields were transformed into discrete values (categorical variables) that can be used for the analysis. In addition, the names were also transformed in order not to reveal information about the possible meanings of the values.

Table I groups the 9 categorical variables and roughly explains the meaning of each group. Table II outlines basic statistics on each of the variables.

TABLE I. ROUGH GROUPING OF THE 9 CATEGORICAL VARIABLES

\begin{tabular}{|l|l|l|l|}
\hline Group & tab & Relates to & Example \\
\hline A & 3 & Scheduling information & $\begin{array}{l}\text { Resources } \\
\text { requested by } \\
\text { the job }\end{array}$ \\
\hline B & 2 & $\begin{array}{l}\text { Execution-specific } \\
\text { information }\end{array}$ & $\begin{array}{l}\text { Command } \\
\text { line and } \\
\text { arguments }\end{array}$ \\
\hline C & 4 & Association information & $\begin{array}{l}\text { Project and } \\
\text { component }\end{array}$ \\
\hline
\end{tabular}

TABLE II. Statistics Regarding the CATEgorical VARIABles

\begin{tabular}{|l|l|l|}
\hline Variable & \# of categories & $\begin{array}{l}\text { \# of missing (in } \\
\text { training data) }\end{array}$ \\
\hline A1 & 9 & 0 \\
\hline A2 & 7 & 0 \\
\hline A3 & 5 & 0 \\
\hline B1 & 44 & 173 \\
\hline B2 & 22 & 184 \\
\hline C1 & 2 & 0 \\
\hline C2 & 5 & 239 \\
\hline C3 & 6 & 184 \\
\hline C4 & 32 & 0 \\
\hline
\end{tabular}

In addition to the above, two additional categorical variables were defined, day and hour, based on the three continuous variables in the trace. These variables indicate the day of the week (1 for Sunday to 7 for Saturday) and the hour of the day (0 to 23), the job was submitted, started, and finished executing, respectively. Figure 2 shows the distribution of the respective temporal categorical variables along the timeline axis. As can be seen, during weekdays longer jobs are typically submitted during the morning hours, with occasional peaks in runtime during evening hours. During weekends, peaks in runtime also occur during the afternoon and evening hours.

Figure 3 shows the distribution of the jobs runtime "as-is", and after applying a log base 2 transformation on the runtime. As can be seen in Figure 3a, the vast majority of the jobs are short (the shortest job ran 3.5 seconds), and there are few long ones (the longest job ran for nearly 9 days). This well corresponds to previous observations made on the runtime, describing a phenomenon that characterizes many production workloads [11].

Transforming the runtime to the log scale (Figure 3b) reveals a mixture pattern of two main Gaussian-like distributions (some-times referred to as a "hyper lognormal distribution), with a stretching right tail.

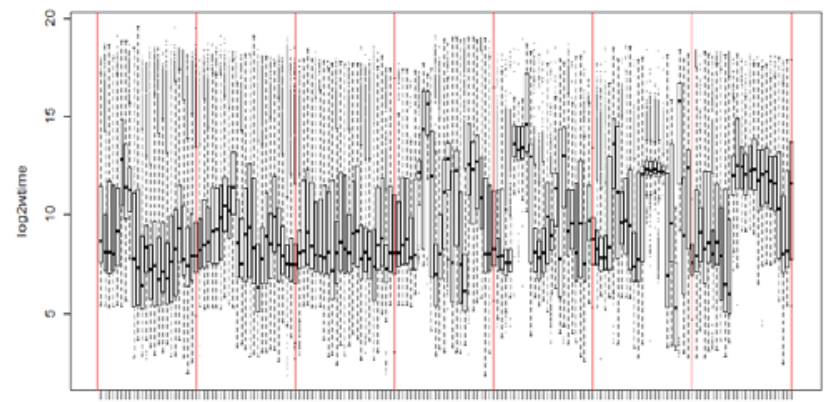

Fig. 2. Runtime boxplots (in log base 2 scale) along time (Sunday through Saturday). The days are separated by vertical red lines. Each tick mark along the time axis marks an hour of the day

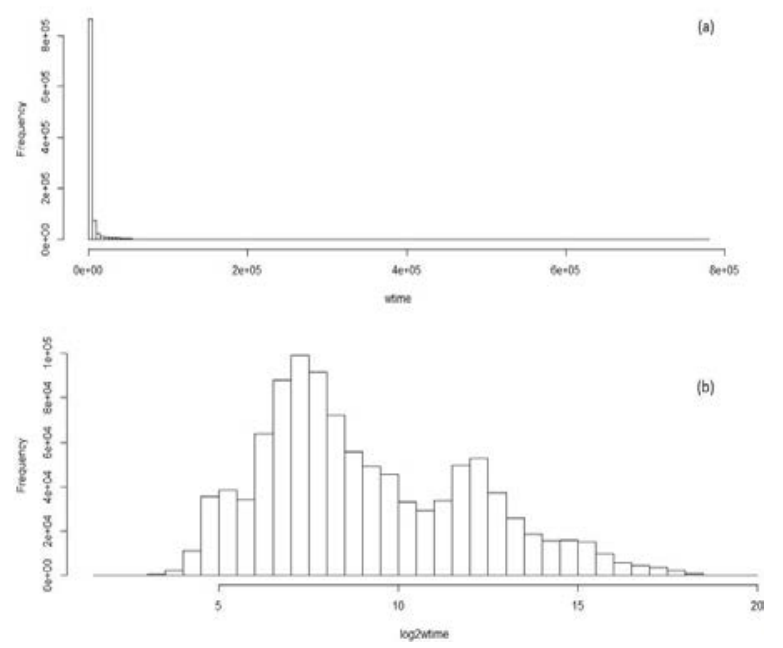

Fig. 3. Histogram of runtime. (a) Raw data (b) After log base 2 transformation 


\section{Class CONSTRUCTION BY MIXTURE DISTRIBUTION ANALYSIS}

The first component in the proposed analysis sets the base for defining the two runtime classes by estimating the mixture distribution parameters, and then labeling each job as "short" or "long". Once the predictor variables are selected through a training-and-testing algorithm (see Section 5.1), the model is optimized by selecting the mixture threshold which provides the best performance, namely minimizes the prediction error or approaches the desired sensitivity-specificity combination (see Section 5.2).

The Gaussian (normal) mixture model has the form

$$
f(x)=\sum_{m=1}^{M} \alpha_{m} \phi\left(x ; \mu_{m}, \Sigma_{m}\right)
$$

with mixing proportions $\alpha_{m}, \sum_{m} \alpha_{m}=1$, and each Gaussian density has a mean $\mu_{m}$ and covariance matrix $\Sigma_{m}$. The parameters are usually fit by the maximum likelihood approach using the EM algorithm, which is a popular tool for simplifying difficult maximum likelihood problems.

Based on the mixture observed in Figure 2, one to four mixture components may be defined. However, for the purpose of the current study it was decided to focus only on two classes, namely "short jobs" and "long jobs". Thus the runtime $Y$ is modeled as a mixture of the two normal variables

$Y$ can be defined by

$$
Y_{1} \sim N\left(\mu_{1}, \sigma_{1}^{2}\right), \quad Y_{2} \sim N\left(\mu_{2}, \sigma_{2}^{2}\right) .
$$

$$
Y=(1-\Delta) \cdot Y_{1}+\Delta \cdot Y_{2}
$$

where $\Delta \in\{0,1\}$ with $\mathbb{P}(\Delta=1)=\pi$. This generative representation is explicit: generate a $\Delta \in\{0,1\}$ with probability $\pi$, and then depending on the outcome, deliver either $Y_{1}$ or $Y_{2}$. Let $\phi_{\theta}(x)$ denote the normal density with parameters $\theta=$ $\left(\mu, \sigma^{2}\right)$. Then the density of $Y$ is

$$
g_{Y}(y)=(1-\pi) \phi_{\theta_{1}}(y)+\pi \phi_{\theta_{2}}(y) .
$$

Suppose the model is fit to the data by maximum likelihood. The parameters are

$$
\theta=\left(\pi, \theta_{1}, \theta_{2}\right)=\left(\pi, \mu_{1}, \sigma_{1}^{2}, \mu_{2}, \sigma_{2}^{2}\right) .
$$

The log-likelihood based on $N$ training cases is

$$
l(\theta ; \mathrm{Z})=\sum_{i=1}^{N} \log \left[(1-\pi) \phi_{\theta_{1}}\left(y_{i}\right)+\pi \phi_{\theta_{2}}\left(y_{i}\right)\right] .
$$

Direct maximization of $l(\theta ; \mathrm{Z})$ is quite difficult numerically due to the sum of terms inside the logarithm. There is, however, a simpler approach. We consider unobserved latent variables $\Delta_{i}$ taking values 0 or 1 as earlier: if $\Delta_{i}=1$ then $Y_{i}$ comes from distribution 2, otherwise $Y_{i}$ comes from distribution 1. Suppose the values of the $\Delta_{i}$ 's are known. Then the log-likelihood would be

$$
\begin{aligned}
l(\theta ; \mathrm{Z}, \Delta)=\sum_{i=1}^{N}[ & \left.\left(1-\Delta_{i}\right) \log \phi_{\theta_{1}}\left(y_{i}\right)+\Delta_{i} \log \phi_{\theta_{2}}\left(y_{i}\right)\right] \\
& +\sum_{i=1}^{N}\left[\left(1-\Delta_{i}\right) \log \pi+\Delta_{i} \log (1-\pi)\right]
\end{aligned}
$$

and the maximum likelihood estimates of $\mu_{1}$ and $\sigma_{1}^{2}$ would be the sample mean and the sample variance of the observations with $\Delta_{i}=0$. Similarly, the estimates for $\mu_{2}$ and $\sigma_{2}^{2}$ would be the sample mean and the sample variance of the observations with $\Delta_{i}=1$.

Since the $\Delta_{i}$ values are actually unknown, the procedure continues in an iterative fashion, substituting for each $\Delta_{i}$ in the previous equation its expected value

$$
\gamma_{i}(\theta)=\mathbb{E}\left(\Delta_{i} \mid \theta, \mathrm{Z}\right)=\mathbb{P}\left(\Delta_{i}=1 \mid \theta, \mathrm{Z}\right),
$$

which is also called the responsibility of model 2 for observation $i$.

We use the following procedure, known as the EM algorithm, for the two-component Gaussian mixture:

1) Take initial guesses for the parameters $\hat{\pi}, \hat{\mu}_{1}, \hat{\sigma}_{1}^{2}, \hat{\mu}_{2}, \hat{\sigma}_{2}^{2}$ (see below).

2) Expectation step: compute the responsibilities

$$
\begin{aligned}
& \hat{\gamma}_{i}=\frac{\hat{\pi} \phi_{\widehat{\theta}_{2}}\left(y_{i}\right)}{(1-\hat{\pi}) \phi_{\widehat{\theta}_{1}}\left(y_{i}\right)+\hat{\pi} \phi_{\theta_{2}}\left(y_{i}\right)^{\prime}}, \\
& i=1,2, \ldots, N .
\end{aligned}
$$

3) Maximization step: compute the weighted means and variances,

$$
\begin{aligned}
\hat{\mu}_{1}=\frac{\sum_{i=1}^{N}\left(1-\hat{\gamma}_{i}\right) y_{i}}{\sum_{i=1}^{N}\left(1-\hat{\gamma}_{i}\right)}, & \hat{\sigma}_{1}^{2}=\frac{\sum_{i=1}^{N}\left(1-\hat{\gamma}_{i}\right)\left(y_{i}-\hat{\mu}_{1}\right)^{2}}{\sum_{i=1}^{N}\left(1-\hat{\gamma}_{i}\right)}, \\
\hat{\mu}_{2}=\frac{\sum_{i=1}^{N} \hat{\gamma}_{i} y_{i}}{\sum_{i=1}^{N} \hat{\gamma}_{i}}, & \hat{\sigma}_{2}^{2}=\frac{\sum_{i=1}^{N} \hat{\gamma}_{i}\left(y_{i}-\hat{\mu}_{1}\right)^{2}}{\sum_{i=1}^{N} \hat{\gamma}_{i}},
\end{aligned}
$$

and the mixing probability,

$$
\hat{\pi}=\frac{\sum_{i=1}^{N} \hat{\gamma}_{i}}{N}
$$

4) Iterate steps 2 and 3 until convergence.

In the expectation step, a "soft" assignment of each observation to each model is done: the current estimates of the parameters are used to assign responsibilities according to the relative density of the training points under each model. In the maximization step, the responsibilities are used within weighted maximum-likelihood fits to update the estimates of the parameters.

A simple choice for initial guesses for $\hat{\mu}_{1}$ and $\hat{\mu}_{2}$ is two randomly selected observations $y_{i}$. The overall sample variance $\sum_{i=1}^{N} \frac{\left(y_{i}-\bar{y}\right)^{2}}{N}$ can be used as an initial guess for both $\hat{\sigma}_{1}^{2}$ and $\hat{\sigma}_{2}^{2}$. The initial mixing proportion $\hat{\pi}$ can be set to 0.5 .

The "mixtools" $\mathrm{R}$ package [15, 16] was used for the mixture analysis, with the function "normalmixEM" for parameter and posterior probability (responsibility) estimation.

\section{THE CART MODEL}

The CART (Classification and Regression Trees) model, also named the decision tree model, is an approach for making either quantitative or class prediction. The CART model is non-parametric, thus no assumptions are made regarding the underlying distribution of the predictor variables, enabling CART to handle numerical data that are skewed or multi- 
modal. Both continuous and categorical predictors can be considered, including ordinal ones.

CART identifies classifying, or "splitting", variables based on an exhaustive search of all classifying possibilities with the available variables. Useful CART trees can be generated even when there are missing values for some variables, by using "surrogate" variables, which contain information similar to the missing variables.

CART analysis consists of the following steps:

- Tree building, during which a tree is built using recursive splitting of nodes. This process stops when a maximal tree has been produced. The higher the splitter variable in the tree, the higher its importance in the prediction process.

- Tree "pruning", which is a simplification of the tree by cutting nodes off from the maximal tree.

- Optimal tree selection, which selects one tree from the set of pruned trees with the least evidence of over fit.

Each path from the root of a decision tree to one of its leaves can be transformed into a rule. Less complex decision trees are preferred, since they are easier for interpretation and may be more accurate.

\section{MODEL LEARNING AND OPTIMIZATION PROCEDURE}

Once labeled data is obtained, a supervised learning technique is used for the purpose of generating a classification rule. In the first stage a set of variables that will be included in the model are selected, while evaluating the performance of each model, and in the second stage a final model is obtained by using the selected variables on the full dataset and evaluate the model based on the performance target function.

This stage of the analysis is done on a subset of the training data (containing the ten days period), which is extracted as follows. Since the two observed runtime distributions overlap, observations that are within 0.5 standard deviations off the two means are selected, such that they will be distant from the overlapping region and will belong to the corresponding classes with high certainty (Figure 4). A total of 257,467 observations labeled short $=1$ (belonging to the Gaussian population with the smaller mean) and 192,205 observations with short $=0$ (belonging to the Gaussian population with the larger mean) were selected. Together they made around $43 \%$ of the data.

A five-fold cross-validation procedure is then performed in order to select variables and evaluate each model, by iteratively dividing the data in random into a training set $(80 \%$ of the learning data) and an evaluation set (20\% of the learning data) and implementing the CART model with the mixture threshold of 0.5. The importance measure, which considers how high the splitting variable is in the tree, is averaged across all iterations for each variable, and the variable having an importance score above the baseline level is selected.

Once a set of variables is selected for each model type, a model is fit to the full training data. We account for the two types of misclassification error, the false "positive" classification and the false "negative" classification. Defining classification into "short" as "positive", the former refers to the erroneous classification of a long job into the "short" class, and the latter refers to the erroneous classification of a short job into the "long" class.

In the job runtime context, sensitivity is defined as the proportion of short jobs classified as short, while the specificity is defined as the proportion of long jobs classified as long. Subtracting the specificity from 1 will give the proportion of long jobs erroneously classified as short. For the CART classifier, the mixture threshold that yields the best tradeoff between the two errors is chosen. The full set of sensitivityspecificity combinations can be summarized in a pseudo-ROC (Receiver Operating Characteristic) curve, in which the sensitivity is plotted against 1-specificity, for each threshold of the probability obtained in the mixture model (the final value obtained for equation 1).

Log2(wime) histogram with labeled observations

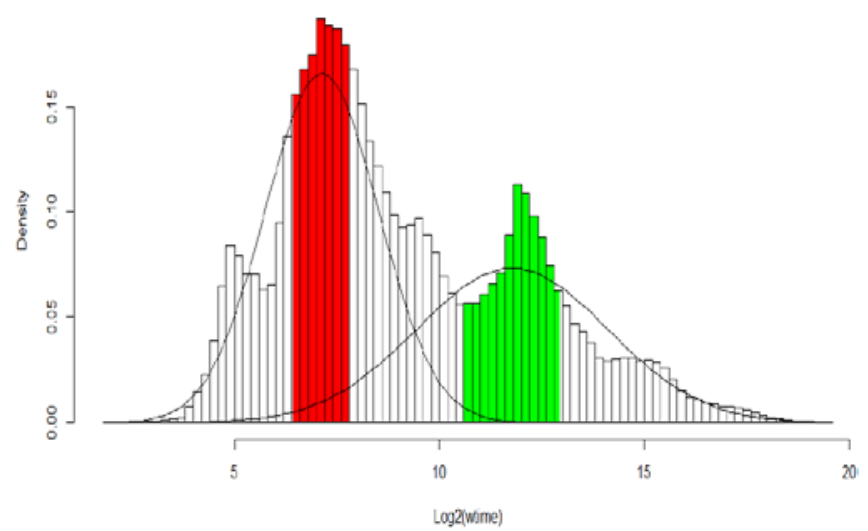

Fig. 4. Runtime (in log base 2 scale) density. The red and green colored regions mark the observations selected for the learning process

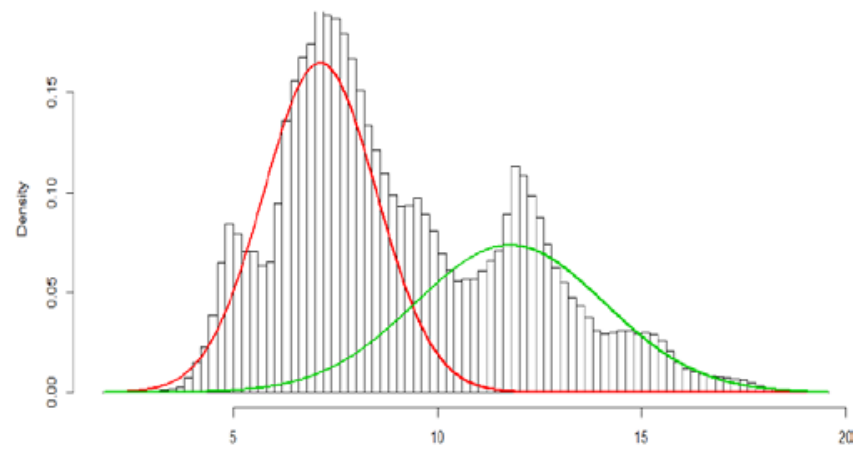

Fig. 5. Density estimates obtained by mixture analysis for the two families underlying the runtime distribution (on the log base 2 scale). The red line marks the estimated density for the "short" class, while the green line marks the estimated density for the "long" class

A model performing a perfect discrimination has an ROC curve that passes through the upper left corner (100\% sensitivity, $100 \%$ specificity). Therefore the closer the ROC curve to the upper left corner, the higher the overall accuracy of the test. Yet, the consequences, or costs, of each type of error may vary among applications and among policy makers. Thus the optimal threshold may allow higher weight to one of the errors on account of the other. 


\section{RESUlTS}

\section{A. Class definition}

Implementing the mixture model clustering approach, two Gaussian families underlying the runtime distribution (on the log base 2 scale) were defined. The density estimates are super imposed on the runtime density in Figure 5. The parameters for each family and the mixing proportions are detailed in Table III.

The mixture analysis also yields the posterior probability, as defined by equation (1), for each observation to belong to the "short" class. For a probability threshold of 0.5, 631,059 observations (nearly 60\%) are classified into the "short" class, while 411,053 observations are classified into the "long" class. Once a classifier is found (see the next subsection), the threshold is refined to optimize the sensitivity-specificity tradeoff.

\section{B. Classifying by CART}

Applying the CART classifier on the training data through the cross-validation procedure, six variables obtained high importance scores (Figure 6). The classifier achieved a total misclassification error of $3.5 \%$.

TABLE III. DENSITY PARAMETER ESTIMATES OBTAINED BY THE MIXTURE ANALYSIS

\begin{tabular}{|l|c|c|c|c|}
\hline & \multicolumn{2}{|l|}{ First Gaussian Family } & \multicolumn{2}{l|}{ Second Gaussian Family } \\
\hline $\begin{array}{l}\text { Mixing } \\
\text { Proportion }\end{array}$ & \multicolumn{2}{|l|}{0.57} & 0.43 \\
\hline Mean & 7.13 & $2^{7.13}$ & 11.78 & $2^{11.78}$ \\
\hline $\begin{array}{l}\text { Standard } \\
\text { Deviation }\end{array}$ & 1.38 & $2^{1.38}=2.60 \mathrm{sec}$ & 2.32 & $2^{2.32}=4.99 \mathrm{sec}$ \\
\hline
\end{tabular}

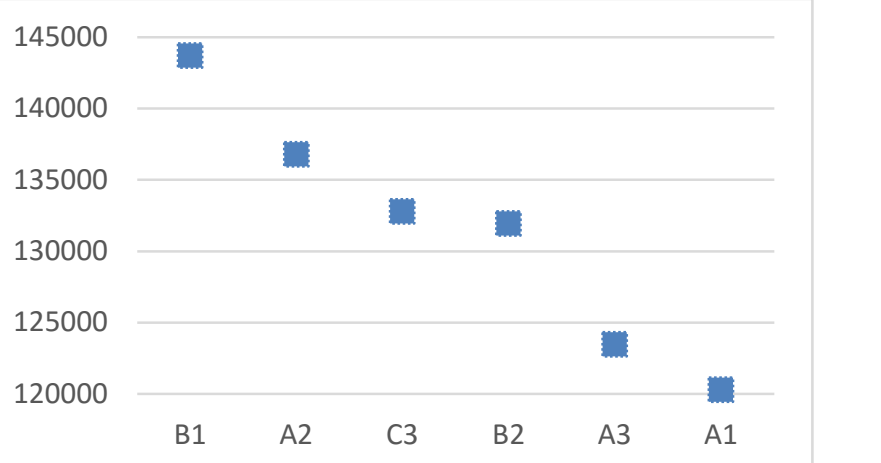

Fig. 6. Top ranking importance scores for the CART model, averaged across 150 cross-validation iterations

A model containing the six selected variables was fit to the full dataset for a series of class mixture threshold. The pseudoROC in Figure 7 presents the sensitivity-specificity combinations obtained for a set of threshold probability used for the mixture distribution. The best performing model is the one using the threshold of 0.45 , which achieves sensitivity of $92.5 \%$ and specificity of $91.1 \%$, with a total misclassification error of $8.9 \%$. This threshold corresponds to a runtime of 9.25 on the log scale, or 608 seconds on the original scale.
The selected model yielded a tree containing four of the six variables that were tried (Figure 8). The total misclassification rate was $8.08 \%$. Implementing the obtained tree on the validation data resulted in a total misclassification error of $9.17 \%$, with specificity of $91.5 \%$ and sensitivity slightly beyond $90 \%$.

\section{RELATED WORK}

Supplying the scheduler with information on how long the jobs are expected to run has always been a challenging task. In general, two approaches were used estimating runtime. The first is to ask the users to supply the information, and the other is to try and predict the runtimes automatically using historical data on jobs that have already completed.

Asking the users to estimate the runtimes has been shown to be highly inaccurate, as users tend of overestimate the runtimes in order to prevent the scheduler from killing their jobs [1]. Furthermore, Tsafrir et al. [2] has observed that the users further tend to "round" the estimates, thereby limiting the scheduler's ability to optimize the schedule. Bailey et al. [7] have shown that users are quite confident of their estimates, and that most likely they will not be able to improve much the accuracy of their estimates.

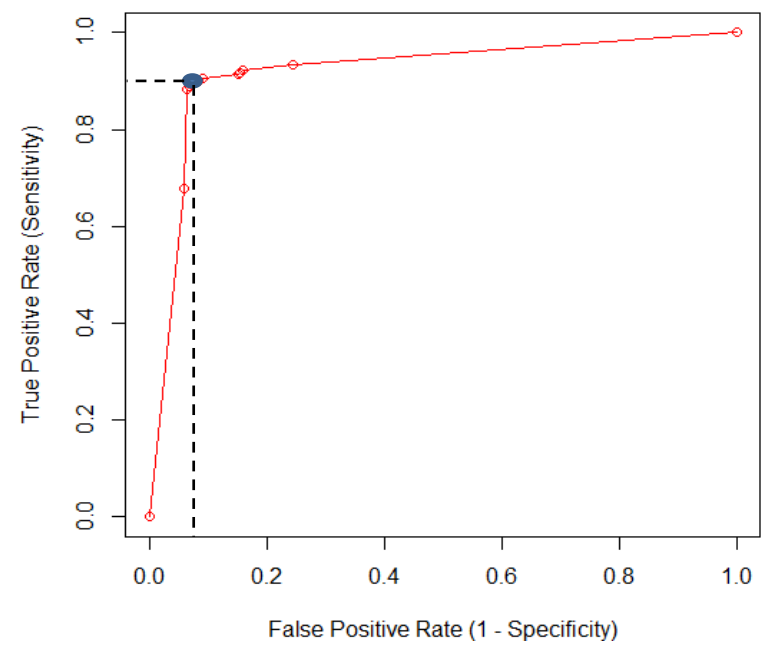

Fig. 7. The pseudo-ROC curve obtained by the CART classifier for the full training data. The blue circle marks the optimal tradeoff between sensitivity and specificity (enhanced by the dashed lines), obtained for mixture probability threshold of 0.45

Predicting the runtimes automatically is therefore the default alternative. This is usually composed of two steps:

1) Identifying classes of similar jobs within the historical jobs records, and

2) Using the aforementioned classes to predict the runtimes for newly submitted jobs.

Gibbons [4] and Downey [5] classified the jobs using a statically defined set of attributes, e.g. user, executable, queue, etc. For newly submitted jobs, Gibbons used the 95th percentile of the runtimes in the respective class, while Downey used a statistical model that was based on a log- 
uniform distribution of the runtimes in order to provide the prediction.

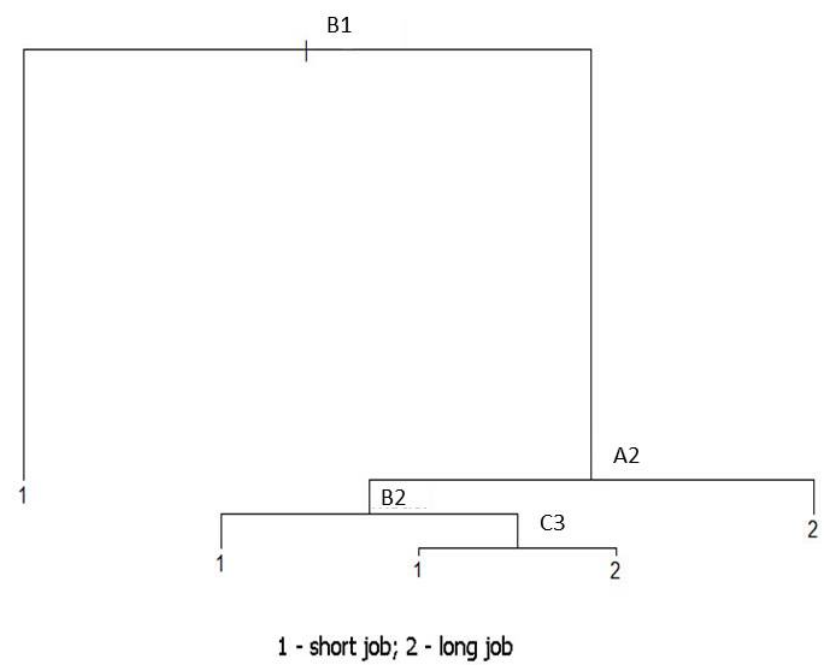

Fig. 8. The final tree obtained on the full data

Smith et al. [6] suggested the use of genetic algorithms to refine the selection of attributes used for the classification, and achieved up to $60 \%$ improvement in accuracy compared to the static approaches. Respectively, Kapadia et al. [8] used instance-based learning and Krishnaswamy et al. [9] applied rough-set theory. Finally, Tsafrir et al. [10] showed that complicated prediction techniques may not be required if the scheduling algorithm itself can be modified, and suggested to average the last two jobs by the same user in the history.

These techniques, however, were mainly designed for parallel systems, in which the scheduler needs to the actual runtimes of the jobs (use numeric predictions) to be able to optimize the schedule. For the server farm usage model which is targeted in the current work, sophisticated scheduling algorithms are not required, and the paper shows that it is enough to simply separate the jobs into short and long in order to improve performance [12]. Our work provides the facility to perform the separation and hence forms the basis for enabling such systems.

\section{DISCUSSION AND CONCLUSIONS}

Predicting the runtimes of jobs using actual numeric values is of high importance for parallel systems. Here, fragmentation is a big concern, and in order to minimize it (namely to fill the holes in the schedule), it is important for the scheduler to know the exact runtime of the jobs. For other types of systems like server farms used for software testing, it is enough to only predict the runtime classes of the jobs e.g., short or long, in order to send the jobs to the right queue and improve performance.

Motivated by the later usage model, a method that facilitates highly accurate prediction of the job runtime class was developed. The method leverages the fact that the runtimes may be represented as a mixture of two or more distributions, in order to train a classifier that will be used to predict the runtime classes of newly incoming jobs. In order to achieve high accuracy, the threshold that separates the short jobs from the long is determined during the evaluation of the classifier. In a real system the threshold can be periodically communicated to the scheduler to help deciding on the right allocation of resources for the different job classes.

The work presented is based on a single data set that was obtained, and included validation on data independent of the training data. Yet, in spite of its promising results, additional testing is required on more data sets in order to establish complete confidence in the robustness of the proposed method. However, due to the size of the data (over one million jobs), and the fact that the mixture distribution is known to be evident in the many real-world workloads, there is a strong reason to believe that with small adjustments e.g., to the number of classes, the proposed method can be tuned to sustain the workloads as well.

A uniqueness of the proposed approach is in conducting an initial step that is aimed to define runtime classes based on the empirical runtime distribution. While other existing approaches for predicting job runtime do not used categorization into classes but rather estimate the runtime numerically, the end result of all approaches is in an overall system efficiency. If efficiency can be defined and measured reliably, it can serve to compare the ultimate performance of all methods. Such assessment is under design and is planned to be conducted as a next step of the presented study.

\section{ACKNOWLEDGMENT}

We thank Prof. Dror G. Feitelson of the Hebrew University, Israel, for the useful comments, Evgeni Korchatov from Intel for helping to obtain and prepare the data and Eran Smadar from Intel for supporting this work.

REFERENCES

[1] Feitelson, D.G., Mu'alem Weil, A.: Utilization and predictability in scheduling the IBM SP2 with backfilling. In 12th IEEE Int'l Parallel Processing Symp. (IPPS), 542-546 (1998)

[2] Tsafrir, D., Etsion, Y., Feitelson, D.G.: Modeling User Runtime Estimates, Job Scheduling Strategies for Parallel Processing (JSSPP), 135 (2005)

[3] Lawson, B.G., Smirni, E., Puiu, D.: Self-Adapting Backfilling Scheduling for Parallel Systems. Proceedings of the 2002 International Conference on Parallel Processing (ICPP), 583-592 (2002)

[4] Gibbons, R.: A Historical Application Profiler for Use by Parallel Schedulers. Job Scheduling Strategies for Parallel Processing (JSSPP), 58-77 (1997)

[5] Downey, A.B.: Predicting Queue Times on Space-Sharing Parallel Computers. Proc. 11th IEEE Int'l Parallel Processing Symp. (IPPS), 209-218 (1997)

[6] Smith, W., Foster, I., Taylor, V.: Predicting application run times using historical information. In the 4th Workshop on Job Scheduling Strategies for Parallel Processing (JSSPP), 122-142, Lect. Notes Comput. Sci. vol. 1459 (1998)

[7] Lee, C.B., Schwartzman, Y., Hardy, J., Snavely, A.: Are user runtime estimates inherently inaccurate? Job Scheduling Strategies for Parallel Processing (JSSPP), 253-263 (2004)

[8] Kapadia, N.H., Fortes, J.A.B., Brodley, C.E.: Predictive ApplicationPerformance Modeling in a Computational Grid Environment. Proc. IEEE Int'l Symp. High Performance Distributed Computing (HPDC), 6 (1999) 
[9] Krishnaswamy, S., Loke, S.W., Zaslavsky, A.: Estimating Computation Times of Data-Intensive Applications. IEEE Distributed Systems Online, vol. 5, no. 4 (2004)

[10] Tsafrir, D., Etsion, Y., Feitelson, D.G.: Backfilling using systemgenerated predictions rather than user runtime estimates. IEEE Trans. Parallel \& Distributed Syst. 18(6), 789-803 (2007)

[11] Feitelson, D.G.: Metrics for mass-count disparity. In 14th Conf. Modeling, Analysis, and Simulation of Comput. and Telecomm. Syst., 61-68 (2006)

[12] Harchol-Balter, M., Crovella, M., Murta, C.: On Choosing a Task Assignment Policy for a Distributed Server System. IEEE Journal of Parallel and Distributed Computing (JPDC), vol. 59, no. 2, 204-228 (1999)

[13] Hastie, T., Tibshirani R., Friedman, J.: The Elements of Statistical Learning: Data Mining, Inference, and Prediction, Springer (2001)
[14] Maimon, O., Rokach, L.: The Data Mining and Knowledge Discovery Handbook, Springer, XXXVI (2005)

[15] Benaglia, T., Chauveau, D., Hunter, D.R., Young, D.S.: Mixtools: An R Package for Analyzing Finite Mixture Models, Journal of Statistical Software, Vol. 32, No. 6, 1-29 (2006)

[16] Benaglia, T., Chauveau, D., Hunter, D.R., Young, D.S, Elmore, R., Hettmansperger, T., Thomas, H., Xuan, F.: Package 'Mixtools' - Tools for Analyzing Finite Mixture Models, Repository CRAN (2014)

[17] Dudoit, S., Fridlyand, J., Speed, T.P.: Comparison of Discrimination Methods for the Classification of Tumors Using Gene Expression Data. Journal of the American Statistical Association Vol. 97, No. 457, 77-87 (2002)

[18] Lewis, R.J.: An Introduction to Classification and Regression Tree (CART) Analysis. Presented at the 2000 Annual Meeting of the Society for Academic Emergency Medicine in San Francisco, California (2000) 